\title{
Sentinel Lymph Node Analysis in Colorectal Cancer Patients Using One-Step Nucleic Acid Amplification in Combination With Fluorescence and Indocyanine Green
}

\author{
Francesco Esposito ${ }^{1}$, Adele Noviello ${ }^{1}$, Nicola Moles ${ }^{1}$, Enrico Coppola Bottazzi ${ }^{1}$, Mario Baiamonte $^{2}$, \\ Ina Macaione ${ }^{3}$, Umberto Ferbo ${ }^{4}$, Maria Lepore $^{4}$, Antonio Miro ${ }^{1}$, Francesco Crafa ${ }^{1}$ \\ ${ }^{1}$ Oncological and General Surgery Unit, St. Giuseppe Moscati Hospital of National Relevance and High Specialty, Avellino; ${ }^{2}$ General and \\ Emergency Surgery Unit, Civico Benfratelli Di Cristina Hospital, Palermo; ${ }^{3}$ Department of Surgical, Oncological and Stomatological \\ Disciplines, University of Palermo, Palermo; ${ }^{4}$ Institute of Pathology, St. Giuseppe Moscati Hospital of National Relevance and High Specialty, \\ Avellino, Italy
}

Purpose: Analysis of the sentinel lymph node (SLN) in colorectal cancer (CRC) patients was proposed for more accurate staging and tailored lymphadenectomy. The aim of this study was to assess the ability to predict lymph node (LN) involvement through analysis of the SLN with a one-step nucleic acid (OSNA) technique in combination with peritumoral injection of indocyanine green (ICG) and near-infrared (NIR) lymphangiography in CRC patients.

Methods: A total of 34 patients were enrolled. Overall, 51 LNs were analyzed with OSNA. LNs of 17 patients (50\%) were examined simultaneously with hematoxylin and eosin (H\&E) and OSNA.

Results: SLN analysis of 17 patients examined with H\&E and OSNA revealed that OSNA had a higher sensitivity ( 1 vs. $0.55)$, higher negative predictive value ( 1 vs. 0.66$)$ and higher accuracy (100\% vs. $76.4 \%)$ in predicting LN involvement. Overall, OSNA showed a sensitivity of 0.69 , specificity of 1 , accuracy of $88.2 \%$, and stage migration of $8.8 \%$. Compared to those who were OSNA (-), OSNA (+) patients had a greater number of LN metastases ( 4.8 vs. $0.16, \mathrm{P}=0.04$ ), higher G3 rate $(44.4 \%$ vs. $4 \%, \mathrm{P}=0.01)$, more advanced stage of disease (stage III: $77.8 \%$ vs. $16 \% ; \mathrm{P}=0.00$ ) and were more rapidly subjected to adjuvant chemotherapy (39.1 days vs. 50.2 days, $\mathrm{P}=0.01$ ).

Conclusion: SLN analysis with OSNA in combination with ICG-NIR lymphangiography is feasible and can detect LN involvement in CRC patients. Furthermore, it allows for more accurate staging reducing the delay between surgery and adjuvant chemotherapy.

Keywords: Colorectal cancer; Sentinel lymph node analysis; One-step nucleic acid; Fluorescence; Indocyanine green

\section{INTRODUCTION}

Colorectal cancer (CRC) is the third most common cancer worldwide and lymph node (LN) status is the most powerful prognostic

Received: June 13,2018 - Accepted: July 21, 2018

Correspondence to: Francesco Esposito, M.D.

Oncological and General Surgery Unit, "St. Giuseppe Moscati" Hospital of National Relevance and High Specialty, Plesso Città Ospedaliera - Contrada Amoretta; 83100 Avellino, Italy

Tel: +39-08-25-20-33-71, Fax: +39-08-25-20-33-71, E-mail: crafa@tiscali.it ORCID code: https://orcid.org/0000-0002-8885-4873

(C) 2019 The Korean Society of Coloproctology

This is an open-access article distributed under the terms of the Creative Commons Attribution NonCommercial License (http://creativecommons.org/licenses/by-nc/4.0) which permits unrestricted noncommercial use, distribution, and reproduction in any medium, provided the original work is properly cited. factor $[1,2]$. After curative treatment, between $30 \%$ and $65 \%$ of patients with CRC will develop recurrent disease [3]. Under-staging for CRC patients is thought to occur in around $10 \%-20 \%$ of patients and the relevant literature shows that examination of only one LN slide using hematoxylin and eosin (H\&E) staining leaves up to $33 \%$ of metastases unidentified [4].

Moreover, some studies have shown that extensive lymphadenectomy is associated with improved prognosis in patients with more advanced stage CRC, even though numerous postoperative complications related to this extensive surgery are described $[5,6]$. On the other hand, organ-preserving surgery without lymphadenectomy has been proposed for early-CRC with encouraging results $[7,8]$.

Among colorectal surgeons and treatments, it is now accepted 
that "one size does not fit all," and there is increasing agreement regarding the need for a more targeted surgery and a tailored lymphadenectomy [9].

The challenge is the careful selection of patients; early CRC disease could benefit from organ-preserving surgery while more advanced stage disease would require tailored lymphadenectomy taking into consideration LN status, the presence of aberrant lymphatic drainage and "skip" metastases.

The sentinel lymph node (SLN) is defined as the first LN in the lymphatic basin that drains the primary tumor, and it has recently been proposed as a strategy for more precise staging in gastrointestinal tumors [10]. Some groups have demonstrated that peritumoral injection of indocyanine green (ICG) with near-infrared (NIR) provides for visualization of LNs and aberrant lymphatic drainage during CRC resection [11]. Furthermore, a novel technique for SLN analysis has been developed: one-step nucleic acid amplification (OSNA; Sysmex Corp., Kobe, Japan) is a rapid mRNA assay that by measuring levels of cytokeratin 19 (CK19) can to identify the presence of metastases and micro-metastases in the LNs within 30-40 minutes of their removal [12].

The aims of this pilot study were to assess the ability to predict LN involvement in patients with CRC using SLN analysis with OSNA in combination with ICG-NIR lymphangiography and to estimate the benefits of the routine use of this technique.

\section{METHODS}

\section{Study design and population}

This cohort study relies on data retrieved from a prospectively maintained database of consecutive patients undergoing elective colorectal resection for adenocarcinoma at St. Giuseppe Moscati Hospital of Avellino, Italy. The database was implemented in 2016 and included preoperative, operative, and postoperative data. In- clusion criteria were any adult patients diagnosed with CRC requiring surgical treatment. Exclusion criteria included participants with an allergy to any ICG. In total, 34 patients were included in this study.

The study was conducted according to the ethical principles of the Institution following the Declaration of Helsinki and was approved by the Institutional Review Board of the St. Giuseppe Moscati Hospital (approval number: 201801). Written informed consent was granted by study participants for publication of this article.

\section{Surgical technique and histopathological evaluation}

All patients underwent colonic preparation within 3 days prior to surgery. Immediately after access to the abdominal cavity by laparotomy or laparoscopy, a flexible endoscope was passed to the tumor site to allow a 1- to 2-mL submucosal injection of ICG (2.5-5 $\mathrm{mg} / \mathrm{mL}$ ) in four points around the tumor. The mesocolic or mesorectal tissue was then examined under NIR illumination (KARL STORZ SE \& Co., Tuttlingen, Germany) and any identifiable LNs were excised and submitted immediately to the pathology department (Fig. 1). The retrieved LNs were staged according to the classification system of the Japanese Society for Cancer of the Colon and Rectum [13]. LNs harvested in this way were cleaned of fat, frozen in liquid nitrogen and stored at $-80^{\circ} \mathrm{C}$ for processing. LNs weighing between $0.05 \mathrm{~g}$ and $0.6 \mathrm{~g}$ or with a cross diameter of less than $8 \mathrm{~mm}$ were exclusively processed using the OSNA technique. LNs weighing more than $0.6 \mathrm{~g}$ or more than $8 \mathrm{~mm}$ in diameter were dissected and analyzed using both the OSNA and $\mathrm{H} \& \mathrm{E}$ methods. In the latter case, LNs were divided at 2-mm intervals, and nonadjacent blocks were alternatively subjected to histopathological examination with $\mathrm{H} \& \mathrm{E}$ or OSNA as described by Yamamoto et al. [14]. For the OSNA assay, larger LNs or slices of smaller LNs were placed in homogenized lysis buffer (Ly-
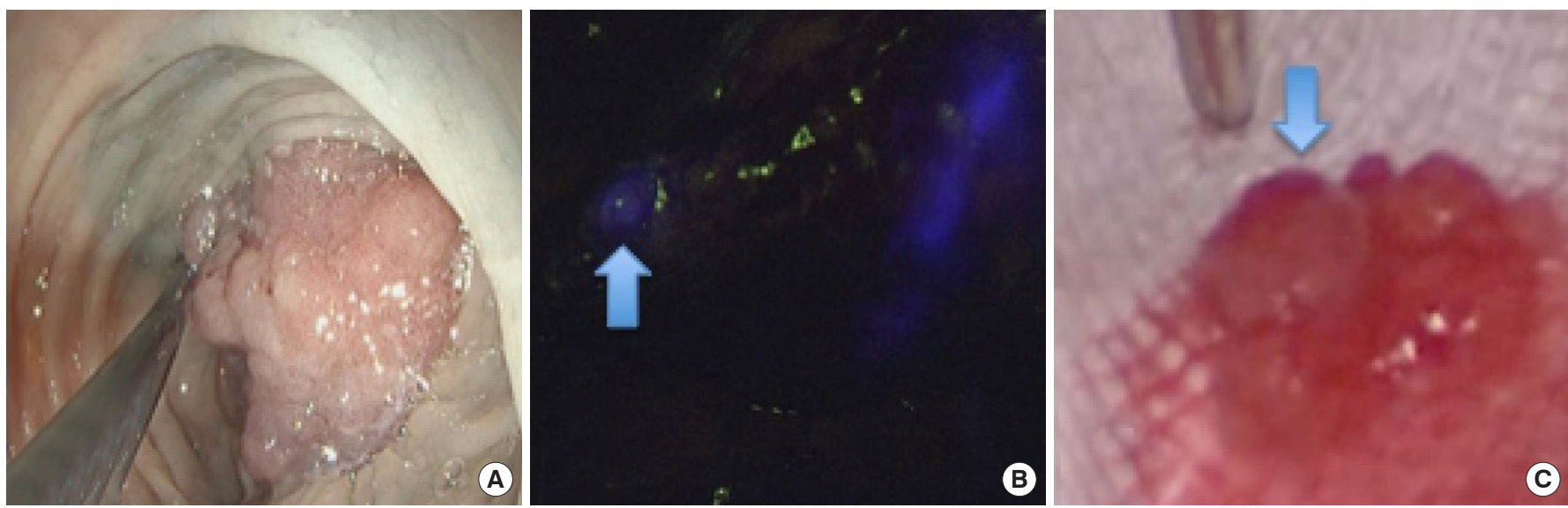

Fig. 1. Procedure for identification of the sentinel lymph node (SLN). (A) Endoscopic submucosal injection of indocyanine green (2.5-5 mg/ $\mathrm{mL}$ ) in 4 points around the tumor. (B) A blue arrow indicates the SLN identified with near-infrared during surgery. (C) A blue arrow indicates the LN harvested and submitted to the pathology department. 


\section{$\begin{aligned} \text { Annals of } & \text { Amplification in Combina } \\ \text { Coloproctology } & \text { Francesco Esposito, et al. }\end{aligned}$}

Table 1. Clinicopathologic characteristics of patients according to OSNA status

\begin{tabular}{|c|c|c|c|c|}
\hline Characteristic & All patients $(n=34)$ & OSNA $(-)(n=25)$ & OSNA $(+)(n=9)$ & P-value \\
\hline Age (yr) & $69.3 \pm 11.8$ & $71 \pm 9.9$ & $64.6 \pm 15.8$ & 0.82 \\
\hline Male sex & $21(61.8)$ & $16(64.0)$ & $5(55.6)$ & 0.70 \\
\hline Body mass index $\left(\mathrm{kg} / \mathrm{m}^{2}\right)$ & $24.8 \pm 2.7$ & $25.1 \pm 2.3$ & $23.9 \pm 3.4$ & 0.33 \\
\hline \multicolumn{5}{|l|}{ Tumor location } \\
\hline Right colon & $10(29.4)$ & $6(24.0)$ & $4(44.4)$ & 0.39 \\
\hline Left colon & $11(32.4)$ & $8(32.0)$ & $3(33.3)$ & 1.00 \\
\hline Rectum & 13 (38.2) & $11(44.0)$ & $2(22.2)$ & 0.42 \\
\hline Neoadjuvant chemotherapy & $2(5.9)$ & $0(0)$ & $2(22.2)$ & 0.06 \\
\hline \multicolumn{5}{|l|}{ Surgical approach } \\
\hline Open & $26(76.5)$ & $17(68.0)$ & $9(100)$ & 0.07 \\
\hline Laparoscopic & 8 (23.5) & $8(32.0)$ & $0(0)$ & \\
\hline \multicolumn{5}{|l|}{ Stadiation } \\
\hline \multicolumn{5}{|l|}{ pT stage } \\
\hline pT1-pT2 & $9(26.5)$ & $9(36.0)$ & $0(0)$ & 0.07 \\
\hline pT3-pT4 & $25(73.5)$ & $16(64.0)$ & $9(100)$ & 0.07 \\
\hline \multicolumn{5}{|l|}{ pN stage } \\
\hline Negative & $21(61.8)$ & $21(84.0)$ & $0(0)$ & $0.00^{*}$ \\
\hline Positive & 13 (38.2) & $4(16.0)$ & $9(100)$ & \\
\hline Sentinel lymph node number examined & $1.5 \pm 0.6$ & $1.5 \pm 0.7$ & $1.3 \pm 0.5$ & 0.31 \\
\hline Total lymph node number & $19.9 \pm 11.1$ & $18.2 \pm 10.2$ & $24.7 \pm 12.9$ & 0.19 \\
\hline Metastatic lymph node number & $1.38 \pm 3.5$ & $0.16 \pm 0.3$ & $4.8 \pm 5.8$ & $0.04^{*}$ \\
\hline$>3$ Metastatic lymph nodes & $3(8.8)$ & $0(0)$ & $3(33.3)$ & $0.01^{*}$ \\
\hline \multicolumn{5}{|l|}{ Lymph node level (JSCCR) } \\
\hline 1 & $12(35.2)$ & $7(28.0)$ & $5(55.6)$ & 0.22 \\
\hline 2 & $11(32.4)$ & $8(32.0)$ & $3(33.3)$ & 1.00 \\
\hline 3 & $11(32.4)$ & $10(40.0)$ & $1(11.1)$ & 0.21 \\
\hline M1 & $2(5.9)$ & $0(0)$ & $2(22.2)$ & 0.06 \\
\hline \multicolumn{5}{|l|}{ Histological grade } \\
\hline G1 (well differentiated) & $1(2.9)$ & $1(4.0)$ & $0(0)$ & 1.00 \\
\hline G2 (moderate differentiated) & $28(82.4)$ & $23(92.0)$ & $5(55.6)$ & $0.03^{*}$ \\
\hline G3 (poorly differentiated) & $5(14.7)$ & $1(4.0)$ & $4(44.4)$ & $0.01^{*}$ \\
\hline \multicolumn{5}{|l|}{ Stage } \\
\hline I & $7(20.6)$ & $7(28.0)$ & $0(0)$ & 0.15 \\
\hline$\|$ & $14(41.2)$ & $14(56.0)$ & $0(0)$ & $0.00^{*}$ \\
\hline III & $11(32.4)$ & $4(16.0)$ & $7(77.8)$ & $0.00^{*}$ \\
\hline IV & $2(5.9)$ & $0(0)$ & $2(22.2)$ & 0.06 \\
\hline Adjuvant chemotherapy & $12(35.3)$ & $4(16.0)$ & $8(88.9)$ & $0.00^{*}$ \\
\hline Time to start adjuvant chemotherapy (day) & $42.8 \pm 6.1$ & $50.2 \pm 4.1$ & $39.1 \pm 1.9$ & $0.01^{*}$ \\
\hline
\end{tabular}

Values are presented as mean \pm standard deviation or number (\%).

OSNA, one-step nucleic acid; JSCCR, Japanese Society for Cancer of the Colon and Rectum.

${ }^{*} \mathrm{P}<0.05$, significant difference. 
norhag, Sysmex) and then centrifuged. Lysate was then extracted for CK19 mRNA RT-LAMP in the RD-100i system (Sysmex) using the Lynoamp (Sysmex) reagent kit [15]. On the basis of previous investigations, OSNA results with CK19 mRNA above 250 copies/L were designated as positive and those with fewer than 250 copies/L were considered negative [12]. The result of the SLN analysis was intraoperatively communicated to the surgeon but the surgical procedure was not modified. All patients underwent standard CRC resection in the conventional manner. A total or partial mesocolon or mesorectal excision was performed. Subsequently, records of each patient were discussed in a multidisciplinary conference with surgeons, pathologists and oncologists, taking into consideration the definitive examination of the surgical specimen and the result of the SLN analysis. The final histology of the surgical specimen and harvested LN were always concluded based on H\&E analysis.

\section{Statistical analysis}

Statistical analyses were carried out by dividing the study population into 2 groups based on the results of the SLN analysis with OSNA. Continuous variables are presented as the mean \pm standard deviations and were compared using Student $t$-test or the nonparametric Mann-Whitney test, as appropriate. Categorical variables are presented as numbers (percentages) and were compared across groups using the chi-square test or Fisher exact test, as appropriate. Furthermore, sensitivity, specificity, positive predictive value (PPV), negative predictive value (NPV), and test accuracy were calculated comparing the intraoperative result of OSNA with the definitive examination of all LNs detected in the surgical specimen, as routinely determined by the pathologist. All statistical analyses were performed with IBM SPSS Statistics ver. 23.0 (IBM Co., Armonk, NY, USA).

Table 2. Comparing definitive histology with OSNA analysis of retrieved fresh lymph nodes also evaluated with hematoxylin and eosin

\begin{tabular}{lccc}
\hline OSNA & Histology $(+)$ & Histology $(-)$ & Total \\
\hline$(+)$ & 9 & 0 & 9 \\
$(-)$ & 0 & 8 & 8 \\
Total & 9 & 8 & 17 \\
\hline
\end{tabular}

OSNA, one-step nucleic acid.

Table 3. Comparing definitive histology with H\&E analysis of retrieved fresh lymph nodes also evaluated with one-step nucleic acid

\begin{tabular}{lccc}
\hline H\&E & Histology $(+)$ & Histology $(-)$ & Total \\
\hline$(+)$ & 5 & 0 & 5 \\
$(-)$ & 4 & 8 & 12 \\
Total & 9 & 8 & 17 \\
\hline
\end{tabular}

H\&E, hematoxylin and eosin.

\section{RESULTS}

\section{Study population}

The characteristics of all patients are shown in Table 1. Thirtyfour patients with colorectal adenocarcinoma were included in this study. Intraoperative evaluation of the SLN with OSNA was positive (OSNA [+]) in 9 patients $(26.5 \%)$ and negative (OSNA $[-])$ in 25 patients $(73.5 \%)$. No complications related to ICG injection occurred during surgery. Globally, 51 LNs were retrieved and examined with a median of 1 (1-3) LN harvested for each patient.

In 17 patients (50\%), the SLN weighed more than $0.6 \mathrm{~g}$ or had a diameter greater than $8 \mathrm{~mm}$, making $\mathrm{LN}$ evaluation possible with both OSNA and H\&E as described. Comparing definitive histology with simultaneous analysis of retrieved fresh LN with OSNA and H\&E, the OSNA technique had higher sensitivity (95\% confidence interval [CI]), $1(0.77-1.00)$ vs. $0.55(0.30-0.78)$, a higher NPV (95\% CI), 1 (0.77-1.00) vs. 0.66 (0.40-0.86), and higher accuracy, $100 \%$ vs. $76.4 \%$. Both methods showed high specificity (95\% CI), $1(0.77-1.00)$ vs. $1(0.77-1.00)$, and a high PPV (95\% CI), 1 (0.77-1.00) vs. 1 (0.77-1.00) (Tables 2 and 3).

Overall, comparing the definitive histology with OSNA analysis of retrieved fresh SLN of all patients, OSNA had a sensitivity (95\% CI) of $0.69(0.51-0.81)$, specificity (95\% CI) of $1(0.87-1.00)$, and accuracy of $88.2 \%$. Its PPV (95\% CI) was $1(0.87-1.00)$, and its NPV (95\% CI) was 0.84 (0.66-0.93) (Table 4). A summary of the sensitivity, specificity, PPV, and NPV are shown in Table 5.

\section{Study OSNA (+) vs. OSNA (-) population characteristics}

OSNA $(+)$ and OSNA $(-)$ patients were statistically similar in age $(\mathrm{P}=0.82)$, sex $(\mathrm{P}=0.70)$, $\mathrm{BMI}(\mathrm{P}=0.33)$, primary disease $(\mathrm{P}=$ 0.39 for right colon, $\mathrm{P}=1.00$ for left colon and $\mathrm{P}=0.42$ for rectal cancer) and the number of patients undergoing neoadjuvant chemotherapy ( $22.2 \%$ vs. $0 \%, \mathrm{P}=0.06$ ) (Table 1$)$. No statistically significant difference was found between the 2 groups in surgical approach, but $100 \%$ of the procedures for patients in the OSNA (+) group were performed by laparotomy $(\mathrm{P}=0.07)$. In $100 \%$ (vs. $16 \%)$ of patients in the OSNA (+) group, $\mathrm{pN}$ was positive on definitive histological examination $(\mathrm{P}=0.00$ ). Globally, $69.2 \%$ (9 of 13) of patients with $\mathrm{pN}$ positive on definitive histological examination were identified during surgery. Yet, a greater number of LNs metastases were found in OSNA $(+)$ patients $(4.8 \pm 5.8$ vs. $0.16 \pm 0.3, \mathrm{P}=0.04$ ). Moreover, $33.3 \%$ (vs. $0 \%$ ) of patients in the

Table 4. Comparing definitive histology with OSNA analysis of all retrieved fresh lymph nodes

\begin{tabular}{lccc}
\hline OSNA & Histology $(+)$ & Histology $(-)$ & Total \\
\hline$(+)$ & 9 & 0 & 9 \\
$(-)$ & 4 & 21 & 25 \\
Total & 13 & 21 & 34 \\
\hline
\end{tabular}

OSNA, one-step nucleic acid. 
Table 5. Summary of sensitivity, specificity, positive predictive value, negative predictive value, and test accuracy

\begin{tabular}{|c|c|c|c|c|c|c|}
\hline Variable & No. & Sensitivity $(95 \% \mathrm{Cl})$ & Specificity $(95 \% \mathrm{Cl})$ & Accuracy (\%) & PPV $(95 \%$ Cl) & NPV (95\% Cl) \\
\hline $\begin{array}{l}\text { Comparing definitive histology with OSNA analysis of SLN } \\
\text { also evaluated with H\&E }\end{array}$ & 17 & $1(0.77-1.00)$ & $1(0.77-1.00)$ & 100 & $1(0.77-1.00)$ & $1(0.77-1.00)$ \\
\hline $\begin{array}{l}\text { Comparing definitive histology with H\&E analysis of SLN } \\
\text { also evaluated with OSNA }\end{array}$ & 17 & $0.55(0.30-0.78)$ & $1(0.77-1.00)$ & 76.4 & $1(0.77-1.00)$ & $0.66(0.40-0.86)$ \\
\hline $\begin{array}{l}\text { Comparing definitive histology with OSNA analysis of all } \\
\text { retrieved SLN }\end{array}$ & 34 & $0.69(0.51-0.81)$ & $1(0.87-1.00)$ & 88.2 & $1(0.87-1.00)$ & $0.84(0.66-0.93)$ \\
\hline
\end{tabular}

Cl, confidence interval; PPV, positive predictive value; NPV, negative predictive value; OSNA, one-step nucleic acid; H\&E, hematoxylin and eosin; SLN, sentinel lymph node.

OSNA (+) group had more than 3 metastatic LNs $(\mathrm{P}=0.01)$. Regarding grading of the tumor, patients with OSNA (-) had a higher rate of G2 (moderately differentiated) (92\% vs. 55.6\%, P = 0.03 ), while patients who were OSNA (+) had a higher G3 (poorly differentiated) rate $(44.4 \%$ vs. $4 \%, \mathrm{P}=0.01)$. A higher number of patients in the OSNA (+) group had distant metastases OSNA (+), though the difference did not reach statistically significance (22.2\% vs. $0 \%, \mathrm{P}=0.06)$. Globally, OSNA (+) patients had more advanced stages of disease (stage III: $77.8 \%$ vs. $16 \%, \mathrm{P}=0.00$; stage IV: $22.2 \%$ vs. $0 \%, \mathrm{P}=0.06)$. After a multidisciplinary discussion, 3 patients $(8.8 \%)$ with SLN positive by the OSNA method were considered $\mathrm{pN}$ positive and subjected to adjuvant chemotherapy. Adjuvant chemotherapy was administered in $88.9 \%$ of patients in the OSNA (+) group (vs. $16 \%, \mathrm{P}=0.00$ ), and the time to start chemotherapy was lower OSNA $(+)(39.1 \pm 1.9$ days vs. $50.2 \pm 4.1$ days in the OSNA [-] group, $\mathrm{P}=0.01)$.

\section{DISCUSSION}

In this study, we showed that intraoperative SLN analysis with OSNA in combination with ICG-NIR lymphangiography is safe, feasible and can predict the state of LN involvement in patients with CRC. Overall, OSNA showed a sensitivity of $69 \%$, specificity of $100 \%$, accuracy of $88.2 \%$, PPV of $100 \%$, negative predictive of $84 \%$, false negative rate of $30.7 \%$ ( 4 of 13 ) and stage migration of $8.8 \%$ (3 of 34 ). Furthermore, $66.7 \%$ (8 of 12 ) of OSNA (+) patients received adjuvant chemotherapy more rapidly.

LN status plays a crucial role in oncologic therapeutic strategies, and despite the use of increasingly sophisticated imaging techniques, pre-operative metastatic $\mathrm{LN}$ identification in patients with CRC is unsatisfactory $[16,17]$.

Innovative organ preserving procedures such as transanal minimally invasive surgery allow an adequate resection of early tumors without resorting to extensive interventions [18]. Nevertheless, it is estimated that $10 \%-20 \%$ of patients in stage T1 will have LN metastasis, and such patients subjected to a localized resection are undertreated [19]. In Japan, on the other hand, unlike in western countries, a lateral $\mathrm{LN}$ dissection in association with conventional total mesorectal excision is performed for advanced rectal cancers (T3-4), and although it is associated with sexual and urinary dysfunction, the risk of intrapelvic recurrence is reduced by $50 \%$, and survival increases $[5,20,21]$.

Particularly in the latter 2 cases, SLN mapping with ICG-NIR lymphangiography may allow the identification of a smaller number of LNs representing the tumor status of the entire nodal basin, directing the surgeon to perform a targeted lymphadenectomy in place of a more radical or conservative treatment. Furthermore, exhaustive analysis of the SLN facilitates a more accurate staging of patients and can influence the decision for adjuvant treatment.

Two results of our study should be highlighted. First, in 3 patients (8.8\%), the SLN analysis was strongly positive by OSNA but negative by $\mathrm{H} \& \mathrm{E}$ evaluation. After multidisciplinary assessment, these patients were considered $\mathrm{pN}$ positive and subjected to migration from stage II to III. This type of approach could lead to more accurate staging of the disease, allowing patients to access adjuvant chemotherapy. In fact, we know that $25 \%$ of CRC patients in stage II will die from recurrent disease [22]. One of the most important risk factors is the presence of unidentified LN micrometastasis. Rahbari et al. [23] concluded in a recent metaanalysis that the presence of LN micrometastasis is associated with poor overall survival and shorter disease-free survival in stage II CRC patients. SLN micro-metastasis can easily be missed by $\mathrm{H} \& \mathrm{E}$ evaluation $[24,25]$. Only immunohistochemistry (IHC) or molecular biology techniques can detect micrometastatic dissemination without doubt, but they are laborious and time-consuming $[25,26]$. OSNA allows detection of the specific mRNA of cytokeratins without classical nucleic acid extraction and purification. Moreover, the analysis of four LNs takes only 40 minutes. Yamamoto et al. [14] found a 95\% concordance rate between OSNA and classical histological analysis with H\&E and IHC. Recently, Miyake et al. [27] demonstrated that the ex vivo analysis of perirectal LNs by OSNA predicts LLN metastasis in advanced rectal cancer with a sensitivity of $100 \%$, specificity of $86 \%$, PPV of $57 \%$ and NPV of $100 \%$. Other studies and a meta-analysis have also confirmed the ability of the OSNA to predict LN involvement [28]. In our study, OSNA was superior to H\&E in identifying LN metastases, with a false negative rate of $0 \%$ vs. $44.4 \%$ and accuracy of $100 \%$ vs. $76.4 \%$, respectively.

Another benefit we found was that OSNA (+) patients received adjuvant chemotherapy more rapidly, an advantage also found by other authors [29]. Indeed, although no definite cutoff has been established, the greater the delay in starting adjuvant chemother- 
apy, the higher the risk of metastatic progression in patients with advanced CRC [30]. When we found LN metastasis during surgery, we planned the placement of a port-a-cath 2 weeks after surgery and started chemotherapy without necessarily waiting for the final pathologic reports.

The limitations of our study are the small number of patients and false-negative rate (30.7\%). We attribute this latter result to the method of lymphangiography rather than OSNA. Emile et al. [31], in a recent meta-analysis that enrolled 12 articles and 248 patients showed a median sensitivity of $73.7 \%$ (range, $0 \%-100 \%$ ), median specificity of $100 \%$ (range, $0 \%-100 \%$ ) and accuracy rates of $75.7 \%$ (range, $0 \%-100 \%$ ) for the detection of metastatic SLN in CRC patients with the use of ICG and NIR combined with H\&E and IHC examination [31]. Except for the higher accuracy rate of our study ( $88.2 \%$ vs. $75.7 \%)$, the remaining results are similar to those reported in this meta-analysis [31]. The site of the ICG injection plays a vital role in determining the outcomes of the test. Emile et al. [31] in effect, have shown that combined subserosal and submucosal injection achieved $100 \%$ rates of sensitivity, specificity and accuracy. Thus far, we have only used submucosal ICG injection just before surgery and will try to modify our technique in order to improve sensitivity. A further limit for the routine use of OSNA is the price, which remains quite high.

In conclusion, SLN analysis with OSNA in combination with ICG-NIR lymphangiography is feasible and may allow intraoperative prediction of LN status in patients with CRC. Furthermore, its implementation allows more precise staging, reducing the delay between surgery and the onset of adjuvant chemotherapy. In our study, the results of SLN analysis did not influence surgical strategy, but OSNA and ICG-NIR lymphangiography could be used to develop customized surgery and tailored lymphadenectomy in patients with CRC.

\section{CONFLICT OF INTEREST}

No potential conflict of interest relevant to this article was reported.

\section{ACKNOWLEDGMENTS}

We are most grateful to Grazia Galoppo for her assistance with data acquisition.

\section{REFERENCES}

1. Goldstein NS. Lymph node recoveries from 2427 pT3 colorectal resection specimens spanning 45 years: recommendations for a minimum number of recovered lymph nodes based on predictive probabilities. Am J Surg Pathol 2002;26:179-89.

2. Ferlay J, Soerjomataram I, Dikshit R, Eser S, Mathers C, Rebelo M, et al. Cancer incidence and mortality worldwide: sources, methods and major patterns in GLOBOCAN 2012. Int J Cancer 2015;
136:E359-86.

3. Pita-Fernández S, Alhayek-Aí M, González-Martín C, LópezCalviño B, Seoane-Pillado T, Pértega-Díaz S. Intensive follow-up strategies improve outcomes in nonmetastatic colorectal cancer patients after curative surgery: a systematic review and metaanalysis. Ann Oncol 2015;26:644-56.

4. Bilchik AJ, DiNome M, Saha S, Turner RR, Wiese D, McCarter M, et al. Prospective multicenter trial of staging adequacy in colon cancer: preliminary results. Arch Surg 2006;141:527-33.

5. Yokoyama S, Takifuji K, Hotta T, Matsuda K, Watanabe T, Mitani Y, et al. Survival benefit of lateral lymph node dissection according to the region of involvement and the number of lateral lymph nodes involved. Surg Today 2014;44:1097-103.

6. Hohenberger W, Weber K, Matzel K, Papadopoulos T, Merkel S. Standardized surgery for colonic cancer: complete mesocolic excision and central ligation--technical notes and outcome. Colorectal Dis 2009;11:354-64.

7. Cahill RA, Leroy J, Marescaux J. Localized resection for colon cancer. Surg Oncol 2009;18:334-42.

8. Creavin B, Ryan E, Martin ST, Hanly A, O'Connell PR, Sheahan K, et al. Organ preservation with local excision or active surveillance following chemoradiotherapy for rectal cancer. Br J Cancer 2017; 116:169-74.

9. Augestad KM, Merok MA, Ignatovic D. Tailored treatment of colorectal cancer: surgical, molecular, and genetic considerations. Clin Med Insights Oncol 2017;11:1179554917690766.

10. Noura S, Ohue M, Seki Y, Tanaka K, Motoori M, Kishi K, et al. Feasibility of a lateral region sentinel node biopsy of lower rectal cancer guided by indocyanine green using a near-infrared camera system. Ann Surg Oncol 2010;17:144-51.

11. Nishigori N, Koyama F, Nakagawa T, Nakamura S, Ueda T, Inoue $\mathrm{T}$, et al. Visualization of lymph/blood flow in laparoscopic colorectal cancer surgery by ICG fluorescence imaging (Lap-IGFI). Ann Surg Oncol 2016;23 Suppl 2:S266-74.

12. Tsujimoto M, Nakabayashi K, Yoshidome K, Kaneko T, Iwase T, Akiyama $\mathrm{F}$, et al. One-step nucleic acid amplification for intraoperative detection of lymph node metastasis in breast cancer patients. Clin Cancer Res 2007;13:4807-16.

13. Watanabe T, Muro K, Ajioka Y, Hashiguchi Y, Ito Y, Saito Y, et al. Japanese Society for Cancer of the Colon and Rectum (JSCCR) guidelines 2016 for the treatment of colorectal cancer. Int J Clin Oncol 2018;23:1-34.

14. Yamamoto H, Sekimoto M, Oya M, Yamamoto N, Konishi F, Sasaki J, et al. OSNA-based novel molecular testing for lymph node metastases in colorectal cancer patients: results from a multicenter clinical performance study in Japan. Ann Surg Oncol 2011; 18:1891-8.

15. Notomi T, Okayama H, Masubuchi H, Yonekawa T, Watanabe K, Amino N, et al. Loop-mediated isothermal amplification of DNA. Nucleic Acids Res 2000;28:E63.

16. Dighe S, Purkayastha S, Swift I, Tekkis PP, Darzi A, A'Hern R, et al. Diagnostic precision of CT in local staging of colon cancers: a 
meta-analysis. Clin Radiol 2010;65:708-19.

17. Choi AH, Nelson RA, Schoellhammer HF, Cho W, Ko M, Arrington A, et al. Accuracy of computed tomography in nodal staging of colon cancer patients. World J Gastrointest Surg 2015;7:11622.

18. deBeche-Adams T, Hassan I, Haggerty S, Stefanidis D. Transanal minimally invasive surgery (TAMIS): a clinical spotlight review. Surg Endosc 2017;31:3791-800.

19. Sakuragi M, Togashi K, Konishi F, Koinuma K, Kawamura Y, Okada M, et al. Predictive factors for lymph node metastasis in T1 stage colorectal carcinomas. Dis Colon Rectum 2003;46:162632.

20. Matsuoka H, Masaki T, Sugiyama M, Atomi Y. Impact of lateral pelvic lymph node dissection on evacuatory and urinary functions following low anterior resection for advanced rectal carcinoma. Langenbecks Arch Surg 2005;390:517-22.

21. Watanabe T, Itabashi M, Shimada Y, Tanaka S, Ito Y, Ajioka Y, et al. Japanese Society for Cancer of the Colon and Rectum (JSCCR) Guidelines 2014 for treatment of colorectal cancer. Int J Clin Oncol 2015;20:207-39.

22. Weitz J, Koch M, Debus J, Höhler T, Galle PR, Büchler MW. Colorectal cancer. Lancet 2005;365:153-65.

23. Rahbari NN, Bork U, Motschall E, Thorlund K, Büchler MW, Koch $\mathrm{M}$, et al. Molecular detection of tumor cells in regional lymph nodes is associated with disease recurrence and poor survival in node-negative colorectal cancer: a systematic review and meta-analysis. J Clin Oncol 2012;30:60-70.

24. van der Zaag ES, Bouma WH, Tanis PJ, Ubbink DT, Bemelman WA, Buskens CJ. Systematic review of sentinel lymph node map- ping procedure in colorectal cancer. Ann Surg Oncol 2012;19: 3449-59.

25. Wang FL, Shen F, Wan DS, Lu ZH, Li LR, Chen G, et al. Ex vivo localization and immunohistochemical detection of sentinel lymph node micrometastasis in patients with colorectal cancer can upgrade tumor staging. Diagn Pathol 2012;7:71.

26. Resch A, Langner C. Lymph node staging in colorectal cancer: old controversies and recent advances. World J Gastroenterol 2013;19: 8515-26.

27. Miyake Y, Mizushima T, Hata T, Takahashi H, Hanada H, Shoji H, et al. Inspection of perirectal lymph nodes by one-step nucleic acid amplification predicts lateral lymph node metastasis in advanced rectal cancer. Ann Surg Oncol 2017;24:3850-6.

28. Wild JB, Iqbal N, Francombe J, Papettas T, Sanders DS, Ramcharan $\mathrm{S}$. Is it time for one-step nucleic acid amplification (OSNA) in colorectal cancer? A systematic review and meta-analysis. Tech Coloproctol 2017;21:693-9.

29. Marhic A, Tremblay JF, Kaci R, André T, Eveno C, Pocard M. Molecular analysis of sentinel lymph node in colon carcinomas by one-step nucleic acid amplification (OSNA) reduces time to adjuvant chemotherapy interval. Dig Liver Dis 2017;49:924-8.

30. Cheung WY, Neville BA, Earle CC. Etiology of delays in the initiation of adjuvant chemotherapy and their impact on outcomes for Stage II and III rectal cancer. Dis Colon Rectum 2009;52:1054-63.

31. Emile SH, Elfeki H, Shalaby M, Sakr A, Sileri P, Laurberg S, et al. Sensitivity and specificity of indocyanine green near-infrared fluorescence imaging in detection of metastatic lymph nodes in colorectal cancer: systematic review and meta-analysis. J Surg Oncol 2017;116:730-40. 